\title{
CONTRIBUTION A L'ÉTUdE DE LA VALEUR PRATIQUE DES SIGNES LAITIERS ET BEURRIERS CHEZ LA VACHE
}

\author{
par A.-M. LEROY,
}

Chef de Travaux de Zootechnie à l'Institut National Agronomique

Directeur de la Station volante d'expérimentation Zootechnique de l'Office Régional du Nord

Parmi les conclusions de M. J.-E. Lucas, rapporteur général du Congrès de I'Alimentation du bétail, organisé par la Société Nationale d'Encouragement à l'Agriculture, le 28 octobre 1925, figurait un vœu en faveur de la création, dans les diverses régions de la France, de stations d'expérimentation zootechnique ayant pour objet de poursuivre l'étude, dans les conditions ordinaires de la pratique, des améliorations à apporter à l'élevage. Grâce à l'initiative de l'Office Régional du Nord, ce vœu a reçu satisfaction, en ce qui concerne la région septentrionale de notre pays. La première station volante créée par ledit office a été placée sous le patronage du Comité Permanent du Contrôle laitier, organe fédératif des syndicats de contrôle constitué par la Société Nationale d'Encouragement à l'Agriculture. Au point de vue technique, elle se trouve rattachée au laboratoire de Zootechnie de l'Institut National Agronomique.

Mise en route en octobre 1926, la station emploie comme personnel un préparateur auxiliaire, ancien élève des cours de contrôle laitier, organisés depuis 1924 à l'Institut National Agronomique. Ce préparateur, installé à proximité d'une grande étable, a pour mission de procéder au contrôle de l'alimentation et à celui de ? : production laitière. D'accord avec le propriétaire de l'exploitation et les vachers de celui-ci, il peut, sur les indications du directeur de la station, M. Leroy, faire varier pendant une période déterminée la ration de quelques animaux convenablement choisis, et comparer la production de ce lot à celle d'un lot formé d'individus constamment tenus au régime alimentaire habituel de l'étable. Des observations nombreuses peuvent être recueillies de la sorte, en ce qui concerne les relations qui existent entre la conformation des animaux et leur valeur laitière et beurrière, déterminée par le contrôle laitier.

La station volante possède la particularité de pouvoir se transporter successivement dans diverses exploitations, ce qui permet à son personnel de poursuivre dans des conditions différentes la solution d'un même problème.

La question de l'appréciation de la valeur laitière et beurrière des vaches d'après l'examen des caractères extérieurs possède une utilité pratique incontestable. Les bons éleveurs savent habituellement distinguer à première vue une médiocre laitière d'une moyenne et surtout d'une bonne bête. Mais il est plus difficile de choisir à bon escient entre une bonne et une très bonne productrice, car iln'est pas rare de constater 
dans les deux cas un développement à peu près égal de la mamelle et des autres caractères laitiers. Parmi ces caractères, certains semblent directement en rapport, d'une manière incontestable,avec l'aptitude recherchée; mais il en existe d'autres dont l'utilité paraît beaucoup plus discutable. La surface de l'écusson, l'écartement des côtes, la longueur de la queue sont notamment dans ce cas.

Si l'on envisage les particularités de conformation, l'on remarque non sans surprise que les opinions émises par les praticiens et les savants zootechniciens qui se sont occupés de cette question sont parfois divergentes.

Nous avons personnellement noté, au cours de nos entretiens avec les éleveurs normands, que la longueur et la largeur de la tête étaient appréciées d'une manière différente par des connaisseurs très expérimentés. Les uns prétendent que la tête longue est l'apanage de la bonne laitière ; les autres recherchent, au contraire, un profil court, bien concave, avec une très grande largeur entre les orbites. Selon ces derniers, la bonne vache doit avoir de la "cervelle " et du "type ", c'est-à-dire posséder au maximum les caractères définis par le standard de la race.

Cependant, les études entreprises dans différents pays depuis que la pratique du contrôle laitier et l'utilisation des mensurations se sont répandues semblent avoir abouti à quelques résultats positifs.

D'après les travaux allemands de Schmidt, de Kronacher, KLeberGER et HITTCHER, il y aurait un rapport entre le poids des animaux et leur production laitière, les meilleures bêtes se rencontrant de préférence parmi les sujets de grand format. Cette conclusion à été confirmée par les travaux de l'américain John Gowen, qui a trouvé qu'il y avait entre le poids et la production un coefficient de corrélation positif égal à 0,35 .

Mais en ce qui concerne les dimensions de la poitrine, les résultats enregistrés semblent moins nets; tandis que les savants allemands Attinger, Stegmann, Kleberger et Kronacher trouvent que la grande profondeur de poitrine doit être regardée comme un signe favorable, leur collègue BoGDANow prétend que cette corrélation est inexistante.

D'après un travail d'origine suisse, publié sous la direction du Professeur Duerst, il existe une corrélation positive faible entre la longueur de poitrine, mesurée depuis le sternum jusqu'à la partie de la dernière côte la plus éloignée de l'épine dorsale

Quant à la largeur de poitrine, les conclusions des auteurs précédents sont nettement contradictoires, en sorte qu'il est assez difficile d'avoir à ce sujet, après la lecture des travaux dont il s'agit, une opinion définie.

La longueur du trone, dont le rapport avee le poids vif est évident, semble nettement s'accroitre avec la production; des chiffres fort démonstratifs à cet égard ont été publiés par SмITH, KRONACHER et tout récemment par Gowes. 
Ajoutons, pour terminer cette revue, que les travaux entrepris en Allemagne dans le but de vérifier la valeur des caractères laitiers indépendants de la conformation n'ont pas fourni de résultats positifs. Les observations d'origine américaine rapportées par GINIEIS, dans son traité sur la connaissance du bétail, relatives aux dimensions de l'écusson, n'ont pas non plus permis de conclure nettement à la valeur de ce signe. Quant aux caractères beurriers, aucune étude d'ensemble ne paraît jusqu'à présent avoir démontré d'une manière irréfutable et définitive leur utilité pratique.

Parmi les travaux les plus intéressants sur la question de l'appréciation des animaux d'après les caractères extérieurs, il convient de citer ici une autre publication de Gowen. Celui-ci a comparé à la production laitière réelle des animaux les notes de mérite attribuées pour les caractères laitiers des mêmes sujets par un nombre déterminé de juges qualifiés. Le degré de corrélation entre l'interprétation des juges et la valeur laitière des sujets n'a pas dépassé $+0,246$, ce qui est remarquablement faible et tout à fait démonstratif de la difficulté qu'éprouvent les connaisseurs dans l'appréciation des facultés de production du bétail.

Frappés par ces résultats, nous avons eu l'idée de procéder à l'étude systématique des rapports qui existent entre les principaux caractères laitiers et beurriers, décrits dans les traités de zootechnie, et les résultats du contrôle des vaches.

$$
\text { ** }
$$

Nous avons eu la bonne fortune de pouvoir profiter des documents accumulés, depuis 1923, par le Syndicat d'Elevage de l'espèce bovine en Seine-et-Oise.

Nous avons choisi particulièrement 3 exploitations soumises au contrôle depuis la création du Syndicat et nous avons procédé d'une manière systématique sur une centaine d'animaux provenant de ces trois troupeaux aux mesures précises d'un certain nombre de caractères, à l'aide de méthodes dont nous donnons plus loin le détail. Toutes ces mesures ont été effestuées par Mlle Revol, préparatrice à la Station Volante, assistée par les contrôleurs laitiers départementaux de Seineet-Oise.

Afin de déterminer la valeur laitière et beurrière de chaque vache, nous avons considéré chacune de ses lactations successives et effectué la correction de ces chiffres pour tenir compte de l'influence de l'âge. L'on sait, en effet, que l'aptitude laitière s'accroit depuis le $1^{\text {er }}$ vêlage jusqu'à l'âge de 7 à 9 ans, puis diminue ensuite. Des travaux nombreux basés sur l'observation des résultats de contrôle de plusieurs milliers d'animaux ont fait connaître dans ses détails cette variation. Nous avons utilisé les chiffres publiés par C.W. TuRner, dans un récent travail concernant la comparaison de taureaux Guernesey (voir indic. bibliogr. 
16) parce que ce document nous a semblé le plus complet de tous ceux parus à ce jour.

Afin d'aboutir à des conclusions nettes, nous avons pris le soin d'enregistrer toutes nos données sur des animaux de race normande pure inscrits au Herd Book normand et aux Livres zootechniques départementaux. Les beaux troupeaux de M. J.-E. LucAs, à Gournaysur-Marne, de M. DE VILLefranche, à Villarceaux et de l'Institut Pasteur à Villepreux, nous ont permis de constituer une collection de sujets suffisamment homogène, dont quelques-uns se trouvaient remarquables par leur excellente conformation et leur aptitude laitière élevée.

\section{**}

Pour mesurer la grandeur des caractères de conformation et des caractères laitiers et beurriers que nous avions l'intention d'étudier, nous avons employé les procédés suivants:

\section{A. Appréciation des caractères de conformation}

Pour effectuer les mesures des principales dimensions corporelles des animaux, nous nous sommes servis de la méthode d'identification photométrique, dite "méthode zoométrique", que nous avons eu l'oscasion de désrire dans cette Revue, au cours de notre série d'articles intitulée "ce que doit savoir un bon Contrôleur laitier et beurrier" (Cf. Ind. bibl. No 12). Rappelons que cette méthode consiste dans la prise simultanée de 2 clichés, l'un représentant l'animal vu par dessus, tandis que l'autre représente le même sujet vu de profil. Toutes les prinsipales mensurations peuvent être obtenues avec une exactitude suffisante par l'étude attentive des documents ainsi réunis..

$1^{\circ}$ Définition et mesure de la surface de poitrine. - Dans le but d'étudier avec précision les rapports entre les dimensions de la poitrine de nos sujets et leurs facultés laitière et beurrière, nous nous sommes servis des procédés habituels de la géométrie descriptive pour dresser la section idéale du corps de chaque individu par un plan perpendiculaire à son plan de symétrie, passant par l'emplacement de la ceinture métrique. Les divisions de cette ceinture, régulièrement espacées, ont permis la construction par points successifs de ce que nous appelons la «courbe de poitrine in de chaque vache.

L'examen des graphiques correspondant à chaque animal montre qu'il existe à ce point de vue entre les divers sujets des différences considérables.

Incontestablement, les vaches les mieux conformées, c'est-à-dire celles dont la vente au boucher est la plus rémunératrice, possèdent des côtes bien arquées se réunissant à la partie supérieure du tronc suivant une courbe en voûte surbaissée, qui rappelle la figure désignée en architecture sous le nom de plein-cintre. 
La question qui nous préoceupait était de savoir si les bonnes laitières se rencontrent de préférence parmi les sujets à côte plate, dont la moitié supérieure de la courbe de poitrine est en forme d'ogive.

$\mathrm{Si}$, comme le croyaient autrefois les praticiens, la bonne vache laitière rappelle par la forme de son corps un ceuf dont le petit bout serait placé à côté de la tête, il est évident que les meilleures productrices doivent se rencontrer de préférence parmi celles dont la courbe de poitrine présente les dimensions minima.

Pour tenter la vérification de cette hypothèse, nous avons mesuré soigneusement, en nous servant d'un planimètre, les surfaces limitées par les courbes de poitrine de chaque sujet, et nous avons confronté les résultats obtenus avee les chiffres de la production laitière.

$2^{\circ}$ Définition et mesure de la surface du bassin. - L'on sait par ailleurs que le grand écartement des hanches est l'une des qualités fondamentales de la bonne vache.

Il paraissait intéressant de rechercher l'existence d'un lien entre la bonne conformation de cette région et l'aptitude laitière. Pour cela, il était indiqué de calculer la surface de l'aire limitée par les pointes des hanches, l'extrémité des trochanters et les extrémités postérieures des coxaux, et de comparer les chiffres ainsi obtenus aux rendements correspondants. C'est ce que nous avons fait.

$3^{\circ}$ Mesure des dimensions de la tête. - Nous étions également curieux de savoir s'il y avait un rapport entre la production laitière et les dimensions de la tête, car les opinions divergentes émises par les praticiens nous avaient vivement frappés, ainsi que nous l'avons dit précédemment. Il a suffi pour cela, de mesurer sur chaque vache la longueur de la tête prise de l'extrémité poștérieure du chignon jusqu'à la limite supérieure du miroir du mufle. Quant à la largeur de la tête, elle a été prise avec un ruban métrique au niveau des orbites.

$4^{\circ}$ Mesure de la longueur et du diamètre de la queue. - Afin de vérifier ensuite s'il fallait attacher de l'importance aux dimensions de la queue, nous avons procédé à la mesure de la longueur de cet appendice, ainsi qu'à celle de son diamètre à l'extrémité.

5o Mesure de l'écartement des côtes. - Enfin, comme l'écartement des deux dernieres côtes est considéré quelquefois comme un indice laitier digne d'intérêt, il a été procédé aux mesures de cet écartement en trois points équidistants placés entre le tiers supérieur et le tiers moyen de la dernière côte. Nous avons fait ensuite pour chaque individu le calcul de la moyenne de ces trois mesures.

\section{B. Appréciation des caractères laitiers}

Parmi les caractères laitiers, nous nous sommes bornés à étudier les fontaines inférieures ou portes du lait, l'épaisseur de la peau mesurée au niveau de la dernière côte, l'épaisseur de la peau mesurée en arrière 
de la mamelle, le nombre de plis en arrière de la mamelle après la traite, la présence de trayons supplémentaires et la surface de l'écusson.

$1^{0}$ Diamètre des fontaines inférieures. - Il est incontestable que l'irrigation du pis est en rapport direct avee l'aptitude à fonctionner de cet organe. Mais pour avoir exactement la mesure de ce rapport, il était indispensable de prendre les dimensions des fontaines de chaque sujet. L'on s'est servi pour cela d'un jeu de petits calibres en buis tourné dont le diamètre variait de millimètre en millimètre.

Pour procéder à une détermination, l'opérateur devait faire entrer successivement chaćun des calibres de la collection dans la fontaine jusqu'à ce qu'il tombe sur un cylindre trop gros pour pouvoir s'enfoncer. Le diamètre du dernier eylindre essayé avant celui-là était pris pour mesure de l'orifice.

Lorsque l'on s'est trouvé en présence de fontaines multiples, l'on a procédé à la mesure du calibre de chaque orifice et calculé le diamètre d'un orifice commun hypothétique ayant la même surface que l'ensemble des autres.

Une courte expérience a montré que des déterminations effectuées à plusieurs reprises sur un même animal par le même opérateur aboutissaient à des résultats parfaitement comparables.

$2^{\circ}$ Mesure de l'épaisseur de la peau. - S'il faut en croire les traités de zootechnie, la peau fine et souple serait l'apanage des grandes laitières. L'appréciation du caractère "souplesse " paraissant assez difficile, nous nous sommes bornés à prendre les mesures de l'épaisseur de la peau.

Ces mesures ont été relevées sans difficulté par le moyen suivant: l'opérateur choisissait sur chaque individu, à la limite entre la dernière côte et le flanc, une dizaine de points repérés à l'avance. En chaque point, il pinçait un repli de peau entre les 2 mors d'un pied à coulisse, muni d'une règle divisée en millimètres et d'un vernier au dixième.

Il suffisait ensuite de calculer la moyenne des dix nombres ainsi recueillis pour apprécier d'une manière suffisamment exacte la grandeur du caractère étudié.

$3^{\circ}$ L'épaisseur de la peau en arrière de la mamelle. - La même méthode a été employée pour la détermination de l'épaisseur de la peau en arrière de la mamelle. Nous nous sommes contentés seulement iei de prendre 5 mesures pour calculer le chiffre moyen.

L'expérience a montré, en effet, que l'épaisseur en cet endroit varie beaucoup moins d'un point à l'autre que celle de la peau des parties latérales du corps.

$4^{0}$ Nombre de plis en arrière de la mamelle après la traite. - Avec de l'esprit d'observation et de la patience, la détermination du nombre de plis en arrière de la mamelle après la traite s'est montrée relativement facile. Des chiffres fort comparables ayant été recueillis à plusieurs 
jours d'intervalle sur le même animal, il a suffi de noter la moyenne de cees différentes déterminations.

50 Présence de trayons supplémentaires. - La présence ou l'absence de trayons supplémentaires - a été scrupuleusement notée sur tous les animaux mis en observation. L'observateur chargé des essais a signalé dans chaque cas le nombre et les dimensions de ces trayons, en employant le langage conventionnel suivant: pas de trayons, trace de trayons, trayons bien développés, trayons très bien développés.

$6^{\circ}$ Surface de l'écusson. - Enfin, dans le but d'étudier la corrélation entre l'aptitude laitière et la forme de l'écusson, nous avons dessiné sur des silhouettes préparées à l'avance, des eroquis aussi précis que possible de l'arrière train de chaque bête.

Pour avoir une idée suffisamment exacte de la superficie relative des écussons, le relevé de ces dessins a été complété par la mesure de leur largeur à un travers de main en dessous de l'extrémité inférieure de la vulve.

\section{Appréciation des caractères beurriers}

Parmi les caractères beurriers, c'est-à-dire ceux qui doivent se trouver en rapport avec le taux plus ou moins élevé en matière grasse du lait, nous avons retenu comme se prêtant le mieux à une détermination quantitative le nombre de papilles de la bouche (d'après le système Renoult et Lizot) et la quantité de cérumen à l'intérieur des oreilles.

a) Papilles buccales. - La détermination du nombre de papilles buccales ne présente aucune difficulté spéciale. L'opérateur a noté séparément avec soin les papilles crênelées et les papilles mousses, lesquelles sont considérées par les auteurs de cette singulière théorie comme des caractères beurriers favorables.

Une note a été attribuée pour ehaque papille. Les papilles crênelées ont été comptées pour 2 points, et les papilles mousses pour un point, en sorte que la note de papilles d'une vache présentant par exemple, 3 papilles crênelées et 7 papilles mousses, s'élevait done à 13 . Nous avons pensé, en adoptant cette convention, qu'il était logique de donner aux papilles crênelées une valeur particulière à cause de leur rareté et de leurs dimensions.

b) Abondance du cérumen. - Afin de comparer la quantité de cérumen secrétée par les différentes vaches, nous nous sommes. arrêtés, après quelques tâtonnements, au mode opératoire suivant:

Des paquets de coton hydrophile préparés à l'avance ont été soigneusement équilibrés, deux par deux, sur les plateaux d'une balance de précision. A l'arrivée dans la vacherie, les deux tampons ont été légèrement imbibés d'éther. Mais tandis que l'un, destiné à servir de témoin, était mis de côté, l'autre était employé à un nettoyage aussi scrupuleux que possible de l'intérieur des oreilles d'un animal. 
De retour au laboratoire, les tampons ont été séchés à l'air et à l'étuve, puis pesés. La différence entre le poids du tampon chargé de cérumen et celui du témoin correspondant constituait la mesure de l'importance de la secrétion auriculaire.

A titre de vérification, les mêmes opérations ont été tentées sur les mêmes vaches, à deux années d'intervalle. Les chiffres obtenus, bien que légèrement différents, se sont montrés-suffisamment rapprochés les uns des autres pour mériter d'être pris en considération.

(A suivre)

\title{
ENQUÊTE SUR LES LAITS VENDUS A LILLE
}

\author{
par Marcel PAGET
}

Maître de Conférences à la Faculté Libre de Médecine et de Pharmacie de Lillé(1).

A l'heure où l'allaitement naturel est de plus en plus délaissé pour l'allaitement artificiel, il m'a paru intéressant d'étudier au point de vue chimique, la composition du lait de vache vendu à Lille, soit par les laitiers ambulants, soit par leurs intermédiaires (les épiciers détaillants).

En vue de cette étude, j'ai donc procédé à l'achat d'échantillons de lait entier (du moins vendu comme tel), prélevés à diverses périodes de l'année dans les différents quartiers de la ville.

Quarante-cinq prélèvements ont été ainsi effectués.

Les recherches analytiques que j'ai poursuivies visèrent surtout la matière grasse, le lactose et les chlorures (la connaissance du taux de ces deux derniers éléments $m$ 'étant, en effet, nécessaire pour l'évaluation de la constante moléculaire simplifiée apparente dont la valeur fournit actuellement la donnée la plus sûre pour l'appréciation du mouillage du'lait.)

A titre documentaire, j'indique les techniques de dosage que j'ai adoptées :

Le dosage de la matière grasse fut opéré à la fois par le procédé Gerber et par une méthode personnelle que j'ai publiée dans l'Union Pharmaceutique d'août 1927 (méthode d'exécution un peu longue, mais d'une précision rigoureuse et qui nécessite le mélange d'un certain volume de lait avec du sulfate de soude anhydre, puis épuisement par l'éther du magma ainsi obtenu. L'opération se faisait dans un lixiviateur Soxhlet).

Le dosage du lactose fut effectué par la méthode de Bertrand, les laits ayant été au préalable déféqués par la méthode Carrez au ferrocyanure de zinc. Quant au taux de chlorures, il fut déterminé tout d'abord par la méthode de Denigès, puis (dans mes dernières analyses) par une technique sûre et rapide que publiera prochainement mon collègue le Professeur Raquet.

(1) Extrait du Journal des Sciences Médicales de Lille, 27 novernbre 1927. 